\title{
Step-gate polysilicon nanowires field effect transistor compatible with CMOS technology for label-free DNA biosensor
}

\author{
G. Wenga, E. Jacques, A-C. Salaün, R. Rogel, L. Pichon and F. Geneste* \\ Institut d'Electronique et de Télécommunications de Rennes (IETR), UMR CNRS 6164, \\ Université de Rennes 1, 35000 Rennes, France, *Sciences Chimiques de Rennes UMR CNRS \\ 6226, Université de Rennes 1, France \\ Contact : asalaun@univ־rennes1.fr
}

Currently, detection of DNA hybridization using fluorescence-based detection technique requires expensive optical systems and complex bioinformatics tools. Hence, the development of new low cost devices that enable direct and highly sensitive detection stimulates a lot of research efforts. Particularly, devices based on silicon nanowires are emerging as ultrasensitive electrical sensors for the direct detection of biological species thanks to their high surface to volume ratio. In this study, we propose innovative devices using step-gate polycrystalline silicon nanowire FET (poly-Si NW FETs), fabricated with simple and low cost fabrication process, and used as ultrasensitive electronic sensor for DNA hybridization. The poly-SiNWs are synthesized using the sidewall spacer formation technique. The detailed fabrication procedure for a step-gate NWFET sensor is described in this paper. Nocomplementary and complementary DNA sequences were clearly discriminated and detection limit to $1 \mathrm{fM}$ range is observed. This first result using this nano-device is promising for the development of low cost and ultrasensitive polysilicon nanowires based DNA sensors compatible with the CMOS technology.

\section{Keywords}


Polycrystalline silicon nanowire FET (poly-Si NW FET); Bio-sensor; DNA hybridization; Spacer formation technique; CMOS compatibility.

\section{Introduction}

DNA detection technology has been developed rapidly due to its extensive application in clinical diagnosis, bioengineering, environmental monitoring, and food science areas. In particular, successful healthcare in the near future will largely rely on a precise diagnosis of a pathological state and knowledge of particular features of the patient relevant for the disease. This "personalized medicine" is expected to yield both better therapeutic results and cost reduction, and is the subject of intense research efforts. In particular, biomarkers have received great interest as tools for early disease detection, diagnosis and treatments in cancer, cardiovascular diseases or even non-invasive prenatal diagnosis. Among these biomarkers, circulating nucleic acids (DNA, mRNA and miRNA) are gaining great attention in several pathologies, because their detection is minimally invasive (blood sample), acceptable to patients, and highly accurate. Much attention has been paid on the development of miniaturized and sensitive detection methods for detection of these nucleic acids. There are several obvious advantages related to the miniaturization of biological sensors: i) reduction of sample and reagent consumption, ii) increase of the sensitivity and specificity, iii) low cost systems requirements, iv) cost-effective disposable chips because of mass production, v) better process control, because of a faster response of the system [Furuberg et al., 2008]. Development of new technologies for biomarkers detection involves both biological and economic challenges. The cost reduction is a major key for the future widespread applications of the devices in biomedical diagnoses that require mass production devices. Low cost and portable devices for rapid analysis of biomarkers may then revolutionize disease diagnosis 
and healthcare. In this study, we propose simple and low-cost silicon nanowires based sensor for specific nucleic acids detection.

Silicon nanowires have recently been developed as transducers for ultrasensitive biosensors and chemical sensors [Lin et al., 2009; Gao et al., 2007; Stern et al., 2007; Patolsky et al., 2006, Wu et al., 2009]. As their surface can be sensitive to charged species combined with their small size and high surface to volume ratio, silicon nanowires (SiNWs) are the subject of intense research activities for high sensitivity sensor fabrication. The highly sensitive detection based on silicon nanowires enable a change in current due to a field-effect, when the analytical molecules bind to specific recognition molecules at the surface of the nanowire [Elfström et al., 2007]. Because SiNWs synthesis can be compatible with the established silicon technology, enormous research efforts to design and develop new generation of high performance biological and chemical sensors by incorporating the SiNWs as the functional sensitive units are performed. SiNWs based sensor integration will allow reliability and lower manufacturing cost, in addition to the advantageous electronic features of embedded detection and signal processing in silicon technology. Many articles have recently reviewed different approaches for silicon nanowire synthesis. The growth of SiNWs can be achieved in a topdown approach, by using sophisticated expensive lithography techniques, or in a bottom-up approach [Patolsky et al., 2005] by self-assembly growth. The latter approach, however, suffers seriously from the difficulty in precisely positioning the device location. It is a major difficulty to the potential device integration and the reliability of the SiNWs-based sensors. In a top-down approach, SiNWs can be prepared using various advanced methods such as ebeam, AFM or deep UV lithography techniques. Excellent electrical properties of monocrystalline silicon nanowires for biosensing have been demonstrated in the literature on Silicon-On-Insulator wafers [Stern et al., 2007; Li et al., 2004; Cui et al., 2001]. The main disadvantage of these methods using advanced lithographic tools with nanometer size 
resolution rests on the high cost generated. Recently, an interesting approach has been demonstrated to synthesize polycrystalline silicon nanowires (poly-SiNWs) using a classical fabrication method commonly used in microelectronic industry: the sidewall spacer formation technique [Hsiao et al., 2009; Lin et al., 2009; Demami et al., 2009, 2011]. Assets of this technological process rest on low cost lithographic tools use, classical silicon technology compatibility and the possibility to get numerous parallel nanowires with precise location on the substrate. Throughout the fabrication, no expensive lithography tools are needed for definition of nano-scale patterns. The detailed fabrication procedures for a step-gate NWFET sensor is described in this paper and we will show that the proposed method, low-cost and simple, is potentially suitable for future practical manufacturing. Here, we demonstrate that the step-gate SiNW FETs can be used as highly sensitive biological sensor for DNA hybridization detection. 25-mer no-complementary and complementary DNA strands will be used as a proof of concept. This first result suggests that this nano-device is promising for the development of low cost and label-free polysilicon nanowires biosensor compatible with the CMOS technology.

\section{Materials and methods}

\subsection{Materials}

3-Aminopropyltriethoxysilane (APTES) and glutaraldehyde (25\%) in aqueous solution were purchased from Sigma-Aldrich. All synthetic oligonucleotides were purchased from EUROFINS MWG including 5-amino-modified DNA probe (NH2-5'-TCA-ATC-TCG-GGAATC-TCA-ATG-TTA-G3'). Two 25-bases sequences are used as targets: complementary target (5'CTAACATTGAGATTCCCGAGATTGA3'), non complementary DNA target (5'TAAAGCCCAGTAAAGTCCCCCACC3'). Fluorescently DNA 5-amino-modified and 3'fluorescein modified DNA (NH2-5'-TCA-ATC-TCG-GGA-ATC-TCA-ATG-TTA-G3'-flu) 
are used as probes. Phosphate buffered solution (PBS) with $\mathrm{pH} 7.4$ was purchased from Sigma-Aldrich.

\subsection{Fabrication of poly SiNW FETs}

Polysilicon nanowires field-effect transistors are fabricated using a 5 masks process. The process described below is CMOS compatible and the maximum temperature process doesn't exceed $600^{\circ} \mathrm{C}$, which means that sensors can be fabricated on silicon substrate as well as on inexpensive glass substrates. N-type transistors with various parallel polysilicon nanowire channels are fabricated using the sidewall spacer method described below. This method is commonly used in submicron scale device silicon technology to insulate device active area. In our case, the spacer at nanometric scale made of polysilicon constitutes the nanowire. This is an alternative way to synthesize SiNWs in a 2D configuration, and it allows the fabrication of parallel SiNWs network over a large area in coplanar structure. The key nanowire fabrication

steps are illustrated on figure 1 . At first, substrate is covered with a $\mathrm{SiO}_{2}$ dielectric film deposited by APCVD (Atmospheric Pressure Chemical Vapor Deposition) at $420^{\circ} \mathrm{C}$. Then, a 100nm thick in-situ doped silicon layer is deposited by LPCVD (Low Pressure Chemical Vapor Deposition) in an amorphous state at $550^{\circ} \mathrm{C}$ and crystallized by thermal annealing under vacuum at $600^{\circ} \mathrm{C}$ during 12 hours. After patterning, a reactive ion etching (RIE) leads to the formation of the step-gate (Fig.1a) covered by a 100nm thick APCVD oxide acting as gate insulator (Fig.1b). After, two layers of polysilicon are successively deposited. The first one is an undoped layer and the second one is a heavily in-situ $\mathrm{N}$-type doped polysilicon layer (Fig.1c). A patterning and a plasma etching are achieved to create an aperture in the gate insulator (Fig.1d). The two layers (undoped polysilicon and heavily doped polysilicon) are successively etched. Accurate control of the polysilicon layers etching rate leads to the formation of nanometric size sidewall spacers used as undoped nanowires (Fig.1e). Source 
and drain regions are made of heavily in-situ $\mathrm{N}$-type doped polysilicon. Then, the polysilicon nanowires are capped with a 70nm thick APCVD oxide. Finally, an aluminum layer is deposited by thermal evaporation (Fig.1f) and patterned to define gate, source and drain contacts (Fig.1g). Fig. 2 shows a SEM image of a polysilicon nanowire obtained by sidewall spacer formation technique.

\subsection{Surface modification for probes immobilization}

Probes immobilization is performed by functionalization of the SiNW capping layer $\left(\mathrm{SiO}_{2}\right)$. Figure 3 describes the procedure used to immobilize the DNA strands onto the nanowires surface. At first, such functionalization of the SiNW devices is carried out using APTES to convert surface silanol groups $(\mathrm{SiOH})$ to amines. The silicon atom in the molecule of APTES will perform a chemical bond with the oxygen of the hydroxyl group. Samples are heated to $100^{\circ} \mathrm{C}$ for one hour to ensure complete drying of the surface to be treated as the APTES reacts rapidly in contact with water, then samples are immersed in an APTES vapor for five minutes and then re-heat to $100^{\circ} \mathrm{C}$ for one hour. After this first step, amino groups $\left(\mathrm{NH}_{2}\right)$ are the terminal units from the surface. Next, Glutaraldehyde is used as a grafting agent for DNA immobilization. Glutaraldehyde binding is achieved through its aldehyde group $(\mathrm{COH})$ that will ensure a chemical bond with the amino group of APTES. For this step, samples are placed in a desiccator under vacuum containing glutaraldehyde in liquid state that evaporates and settles on the samples during $12 \mathrm{~h}$ at room temperature. For probes immobilization, 3'amino group of DNA strands are linked to the aldehyde groups of the linker. A $10 \mu 1$ drop solution of synthetic 400ng/ $\mu$ l DNA probes is deposited onto the NWs for $1 \mathrm{~h}$. The major challenge in surface modification is the control of DNA probes immobilization over functional groups. To check the efficient probes immobilization, fluorescently DNA probes modified with fluorescein are immobilized and fluorescence images are observed. 


\subsection{Target DNA hybridization}

After probes immobilization, a drop with DNA targets is deposited on the nanowires surface to hybridize to the DNA probes. Complementary and no-complementary targets are used, with various concentrations after dilution with PBS solution. After 1h, samples are washed with PBS to remove excess targets and NWFET static electrical characteristics are performed.

\section{Results and discussion}

\subsection{Electrical measurements of the step-gate poly-Si NWFETs}

Static electrical characteristics are collected at room temperature using a HP4155B semiconductor parameter analyzer. Figure 4 presents output $I_{D}-V_{D S}$ and transfer $I_{D}-V_{G S}$ characteristics for the 70nm curvature radius SiNW based n-channel FET. Fig. 4a highlights the field effect modulation of drain current with positive gate voltage, linear and saturation regions are observed. Transfer characteristic (fig.4b) shows current drain switched between 'on' and 'off' state by varying potential of the gate electrode and the switching ratio between 'on' and 'off' state is $\sim 1.7 \times 10^{4}$. Threshold voltage, $\mathrm{V}_{\mathrm{T}}$, and optimum field effect mobility, $\mu_{\mathrm{FE}}$, are determined according to the classical electrical conduction model used for the MOSFET (Metal Oxide Semiconductor Field Effect Transistor). $\mu_{\mathrm{FE}}$ is deduced from the maximum slope of the $I_{D}-V_{G S}$ curve plotted in the saturation mode, and $V_{T}$ is determined by the intercept of the $I_{D}-V_{G S}$ curve in linear mode with the gate axis voltage. The field effect mobility $\left(\sim 60 \mathrm{~cm}^{2} / \mathrm{V} . \mathrm{s}\right)$ and the high threshold voltage $\left(\mathrm{V}_{\mathrm{T}} \approx 7.5 \mathrm{~V}\right)$ values are related to the poor crystalline quality of the channel. Indeed, note that the step-gate NWFET channel is located on the sidewall of the patterned gate (fig.5) which is the lateral seed layer containing a higher defect density than the upper polycrystalline SiNW surface. Electrical properties of polycrystalline silicon are strongly controlled by defects caused by dangling bonds and 
strained bonds within the grain boundaries [Fortunato, 1997]. These grain boundaries and intergranular defects as well as gate insulator/SiNWs interface defects mainly located in the channel region degrade the carrier transport. However, the influence of the gate voltage on drain current shows that the device can be sensitive to charged molecules. In particular, negative charges carried by the phosphate groups of DNA bound onto NW surface will play the role of chemical gates that exert an electric field.

\subsection{Probes immobilization}

Fluorescently DNA probes modified with fluorescein are immobilized on unmodified surface and on functionalized surface. Fluorescence images are observed to check the efficiency of probes immobilization. DNA strands cannot be linked on the nanowire without functionalization and thus the fluorescence was not observed on fig 6-a. The intensity of fluorescence on sample with functionalization suggests effective DNA strands immobilization (fig.6-b)

\subsection{Electrical response after DNA hybridization}

Hybridization phenomenon is detected on electrical characteristics of the SiNWFET. The transfer characteristic of the transistor after probes immobilization is used as reference (see circles in figure 7). Then, we load a drop of a solution containing a complementary DNA targets (concentration 10fM) on the transistor. After incubation and rinsing, we observe (see squares in Figure 7) a shift of the characteristic in the direction of the lower positive voltages (negative shift) due to the detection of negative charges carried by the phosphate groups of the hybridized complementary DNA. Indeed, negative surface charges act as chemical gate that exert an electric field, inducing a cumulative effect with a positive bias voltage in $\mathrm{n}$-channel formation. Since the surface charge acts as an additional gate, current in the n-channel of the NW-FET at fixed positive step-gate bias increases if negative concentration increases on the 
surface. As a result, lower threshold voltage of the $\mathrm{I}_{\mathrm{D}^{-}} \mathrm{V}_{\mathrm{GS}}$ curve of the NWFET is observed. In addition, non-complementary DNA targets have been tested to check specific hybridization. We observed that the electric characteristics remained unchanged, indicating that no additional charges were present on the surface and also that there is no-specific binding of DNA targets on glutaraldehyde.

These preliminary results indicate that specific DNA molecules may be detected using the SiNW technology described in this paper. The detection range and the lowest detectable concentration have been estimated. The relationship between current variation $\left(\Delta I_{D}=I_{D \text { targets }}\right.$ $\mathrm{I}_{\mathrm{D} \text { probes }}$ for $V_{\mathrm{GS}}=8 \mathrm{~V}$ ) and target concentration is plotted on figure 8 , and reveals a logarithmic dependence. Concentration as weak as $1 \mathrm{fM}$ is detected and this first result using innovative step-gate polysilicon nanowires field effect transistor is promising for the development of low cost and ultrasensitive polysilicon nanowires based DNA sensing applications compatible with the CMOS technology.

\section{Conclusions}

The detailed fabrication procedure for a step-gate polysilicon NWFET sensor is described. We demonstrate that this device can be used as biological sensor for detection of DNA hybridization. This sensor has experimentally demonstrated its high sensitivity using synthetic sequences as probes and targets. No-complementary and complementary sequences were clearly discriminated and detection limits to $1 \mathrm{fM}$ is achieved. Thus, the poly-Si NW FET can be an interesting alternative to conventional DNA-hybridization detection because, in a few minutes, they can analyze biomolecular interactions in realtime and in a label-free way. The first results are promising for the development of low cost, label-free and sensitive polysilicon nanowires based DNA sensing applications compatible with the CMOS technology. Fabricate 
a large amount of devices, control the electrical properties and reduce the cost to a reasonable range will be an important issue for using Si NW FET in biomedical applications. Next studies will focus on the sensitivity limitations, in particular on the detection of DNA mutations which is of crucial interest in molecular biology. Particularly, it is a relevant tool for the diagnosis of several diseases, like some types of cancer related to inherited mutations.

\section{Acknowledgments}

This work was supported by the Agence Nationale de la Recherche (France) with the Project Sena (ANR-09JCJC-0072-01).

\section{References}

- Cui, Y., Wei, Q., Park, H., Lieber, C.M., Nanowire nanosensors for highly sensitive and selective detection of biological and chemical species. Science, 2001. 293(5533): p. 1289-1292.

- Demami F., L. Pichon, R. Rogel, A. C. Salaun, Fabrication of polycrystalline silicon nanowires using conventional UV lithography, Mat. Sc. Eng, 6 (2009) 012014

- Demami F., L. Ni, R. Rogel, A.C. Salaun, L. Pichon, Silicon nanowires based resistors as gas sensors, Sensors and Actuators B: Chemical (2011). Available on line at http://dx.doi.org/10.1016/j.snb.2011.04.083 (2011)

- Elfström, N., Juhasz, R., Sychugov, I., Engfeldt, T., Eriksson Karlström, A., Linnros, J., 2007. Nano Lett. 7, 2608-2612

- Fortunato G., Thin Solid Films 296 (1997) 82-90

- Furuberg L., M. Mielnik, A. Gulliksen, L. Solli, I. Johansen, Microsyst Technol (2008) 14:673681

- Gao et al., Silicon Nanowire Arrays for Label-Free Detection of DNA Anal. Chem., 2007, 79 (9), pp 3291-3297

- Hsiao, C.Y., Lin, C.H., Hung, C.H., Su, C.J., Lo, Y.R., Lee, C.C., Lin, H.C., Ko, F.H., Huang, T.Y., Yang, Y.S., 2009. Biosensors and Bioelectronics 24 (2009) 1223-1229.

- $\quad$ Li Z., Chen Y., Li X., Kamins T.I.,Nauka K., Williams R.S., 2004.Nano Lett. 4, 245-247.

- Lin H.C. et al., Biosensors and Bioelectronics 24 3019-3024, 2009

- Patolsky F. Zheng, G. and Lieber C., Analytical Chemistry, 4261-4269, 2006.

- Patolsky F. and Lieber C, Materialstoday, 22-26, 2005.

- Stern, E., Klemic, J.F., Routenberg, D.A.,Wyrembak, P.N., Turner-Evans, D.B., Hamilton, A.D., LaVan, D.A., Fahmy, T.M., Reed, M.A., 2007. Nature 445, 519-522.

- Wu C-C, Ko F-H, Yang Y-S, Hsia D-L, Lee B-S, Su T-S, Biosensors and Bioelectronics 25 (2009) 820-825 


\section{Figure captions}

Figure 1. Key fabrication steps of poly-Si NW FET. (a) Step-gate formation on a Si substrate capped with an oxide layer. (b) The step gate is covered with an APCVD oxide acting as gate dielectric. (c) Two poly-Si layers are deposited by LPCVD technique: the first one (purple color) is an undoped poly-Si and the second one (green color) is an N-type doped poly-Si. (d) Creation of gate contact. (e) Creation of nanowires after plasma etching. (f) Aluminum layer deposition. (g) 3-D view of the final structure of the fabricated device

Figure 2. SEM image of a polysilicon nanowire.

Figure 3. Schematic of poly-Si nanowire surface functionalization of. (1) The APCVD oxide capping the poly-Si NW is coated with APTES. (2) Glutaraldehyde is linked to the amino groups. (3) The DNA probe reacts with the aldehyde groups. (4) Complementary DNA target is hybridized with the DNA probe.

Figure 4: Electrical characterization of poly-Si NWFET: a) output characteristics $I_{D}-V_{D S}$ and b) transfer characteristics $\mathrm{I}_{\mathrm{D}^{-}} \mathrm{V}_{\mathrm{GS}} 70 \mathrm{~nm}$ curvature radius $\mathrm{SiNW}$ based FET transistor (6 nanowires, $\mathrm{L}=5 \mu \mathrm{m})$.

Figure 5: Schematic illustration of structural defects in the core of the polycrystalline silicon NWs and of an increase in negative charges on the nanowire surface resulted from hybridization between complementary DNA target and probe.

Figure 6: (a). Functionalized surface without DNA. (b) Fluorescence observed after DNA 
hybridization on a functionalized surface.

Figure 7: $\mathrm{I}_{\mathrm{D}}-\mathrm{V}_{\mathrm{GS}}$ curves obtained from a functionalized step-gate poly-SiNW transistor (2 nanowires, $\mathrm{L}=3 \mu \mathrm{m}$ ) after probes immobilization and then after hybridization of $10 \mathrm{fM}$ complementary targets. A constant $\mathrm{V}_{\mathrm{DS}}$ is set at $1 \mathrm{~V}$.

Figure 8: Poly-Si NWFET $(\mathrm{L}=3 \mu \mathrm{m})$ drain current shift versus target DNA concentration. The voltage shift is extracted from concentration-dependant $\mathrm{I}_{\mathrm{D}}-\mathrm{V}_{\mathrm{GS}}$ curves at a constant gate voltage $\left(\mathrm{V}_{\mathrm{GS}}=8 \mathrm{~V}\right)$ and drain voltage $\left(\mathrm{V}_{\mathrm{DS}}=1 \mathrm{~V}\right)$. The correlation coefficient of the linear fit is $\mathrm{R}=0.987$. 
Highly in situ doped polysilicon layer (step-gate)

Si substrate

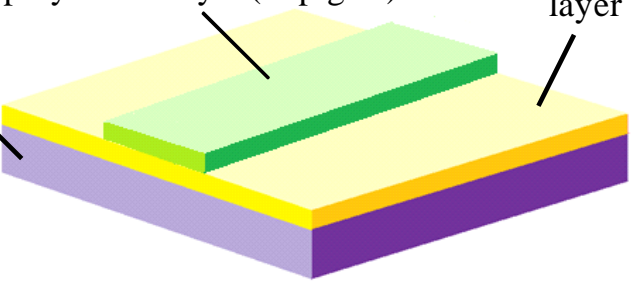

(a)

$\mathrm{SiO}_{2}$ layer

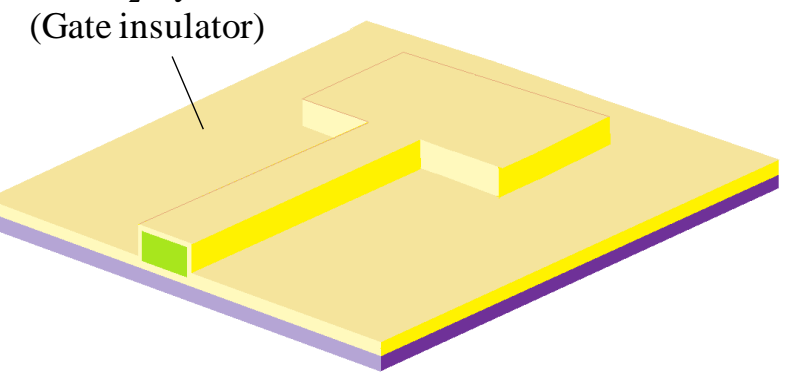

(b)

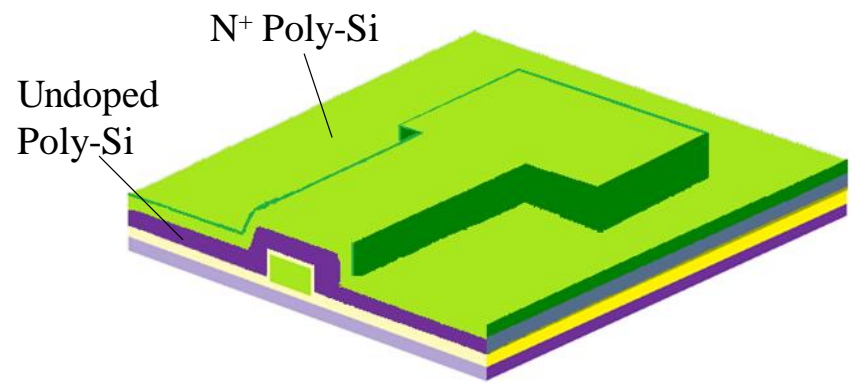

(c)

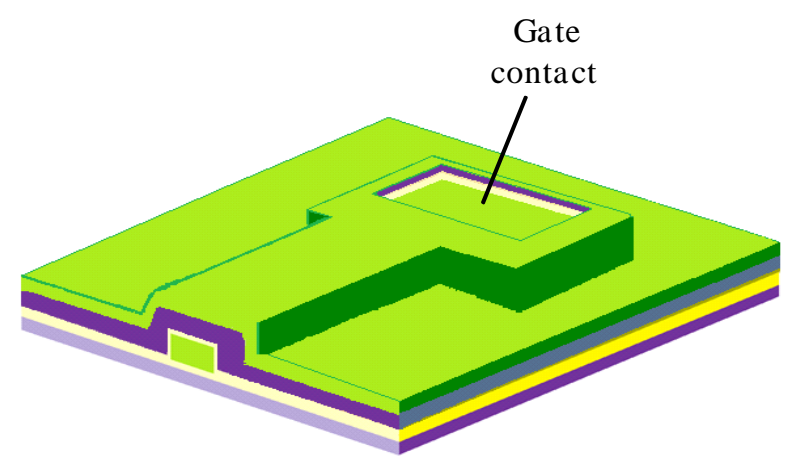

(d)

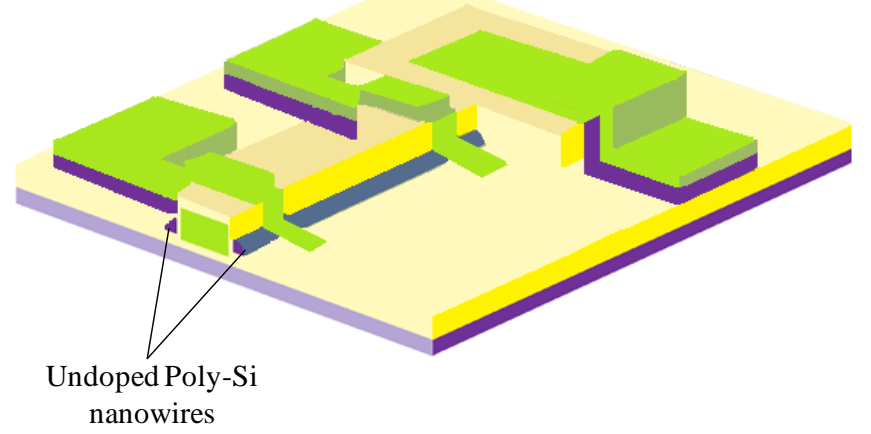

(e)

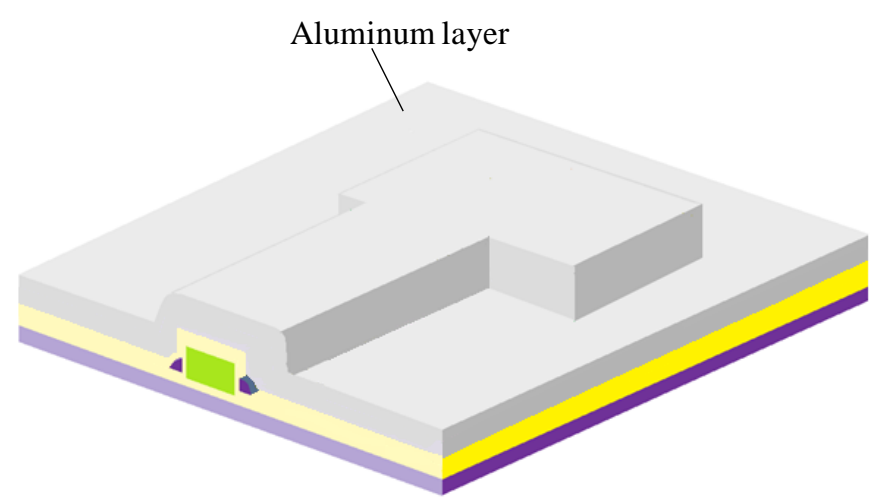

(f)

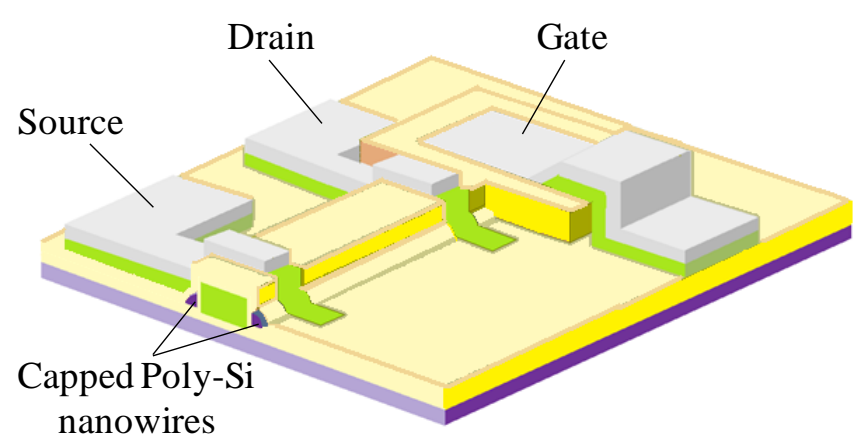

(g)

Fig. 1. 


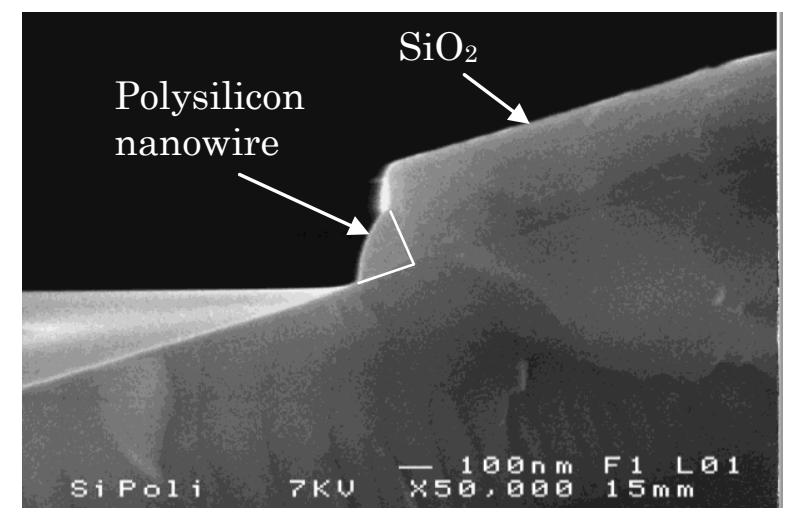

Fig. 2. 

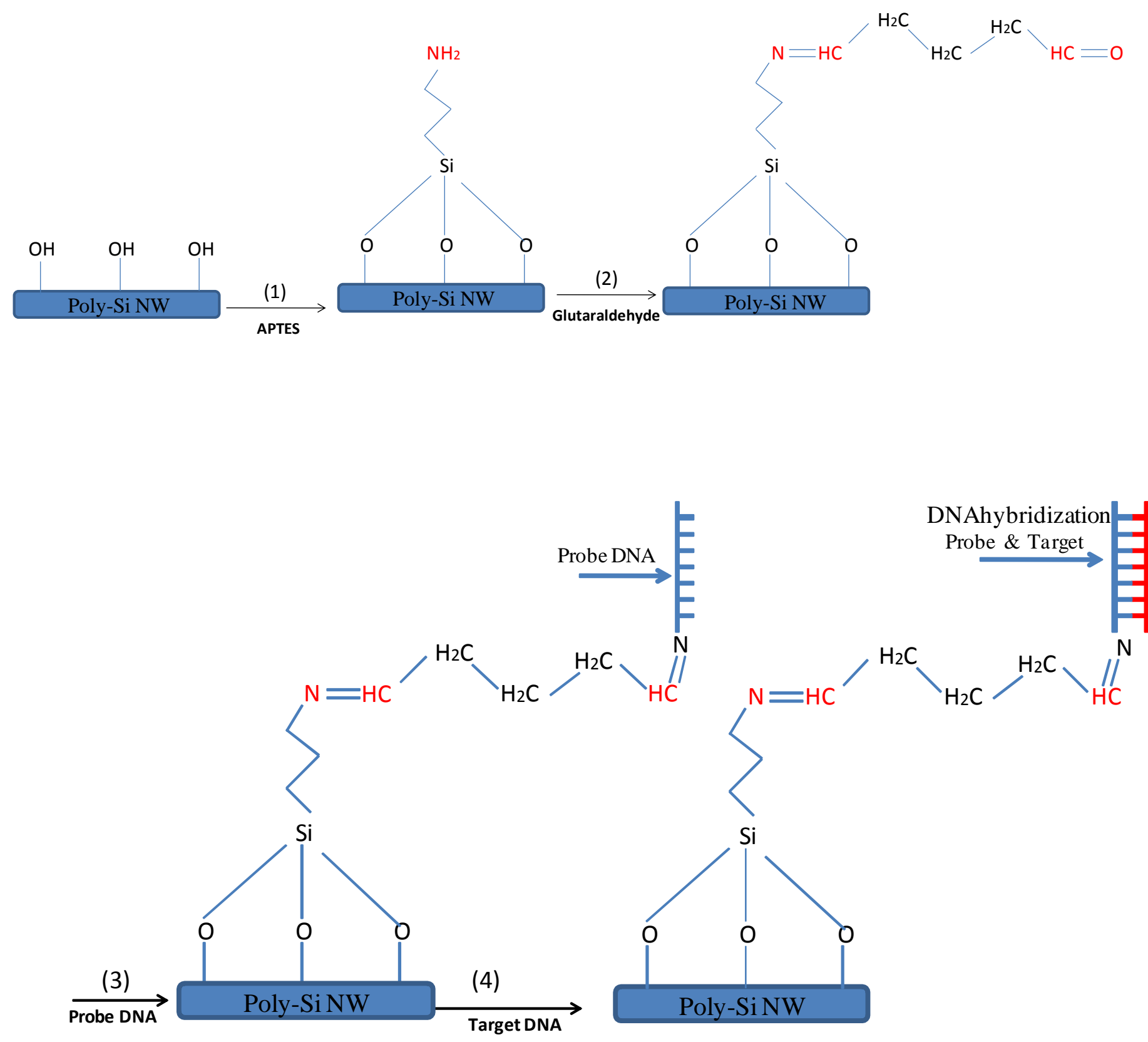

Fig. 3 

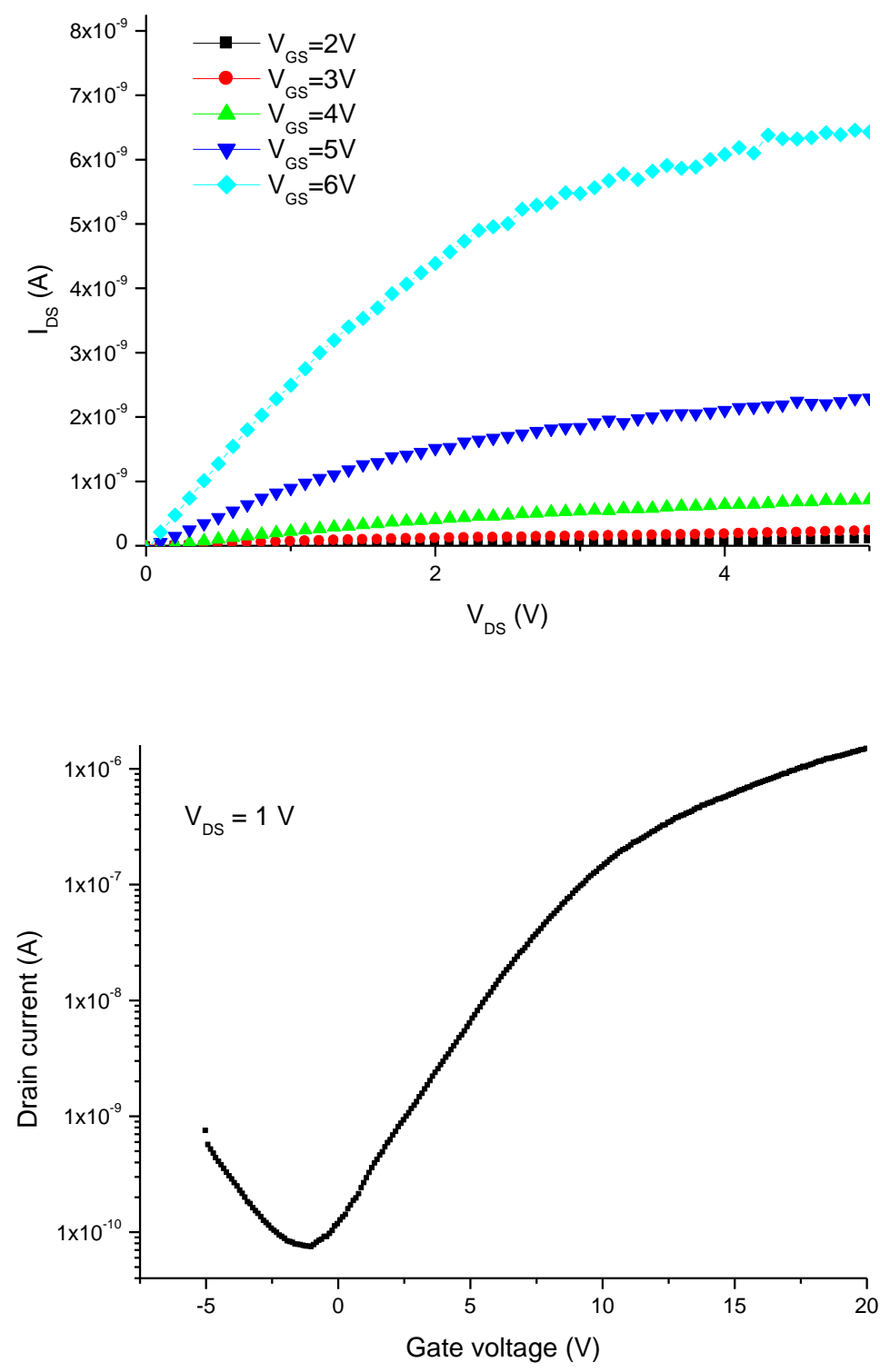

Fig. 4 


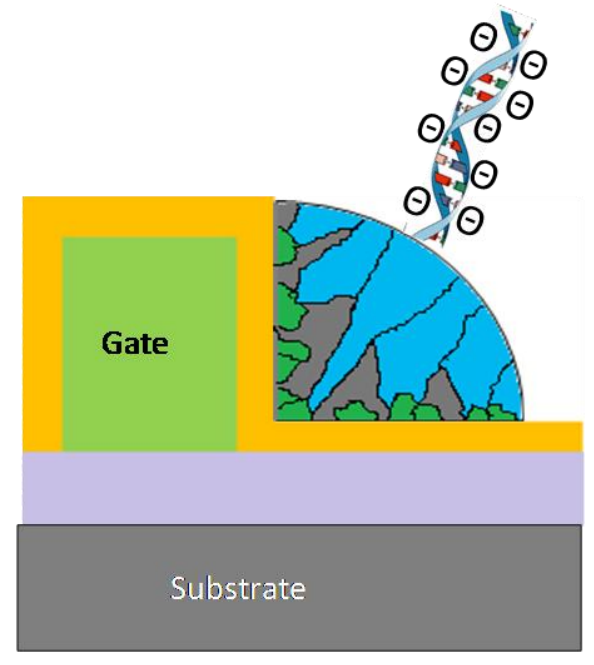

Fig. 5 

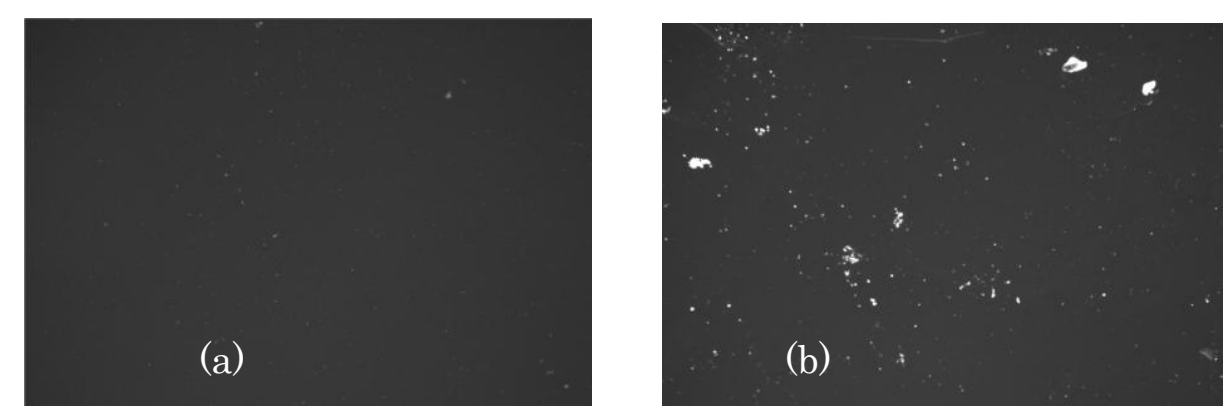

Fig. 6. 


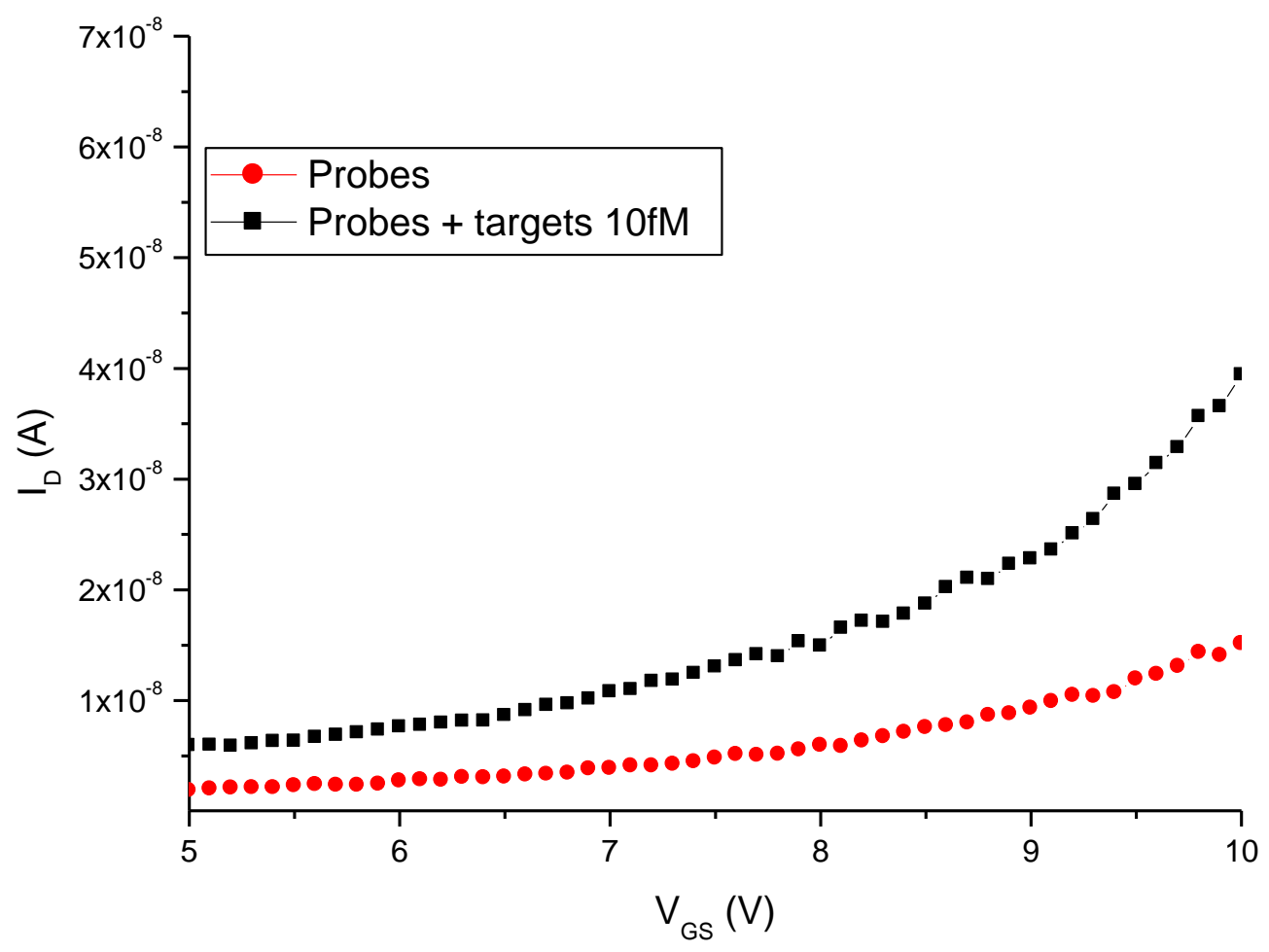

Fig. 7 


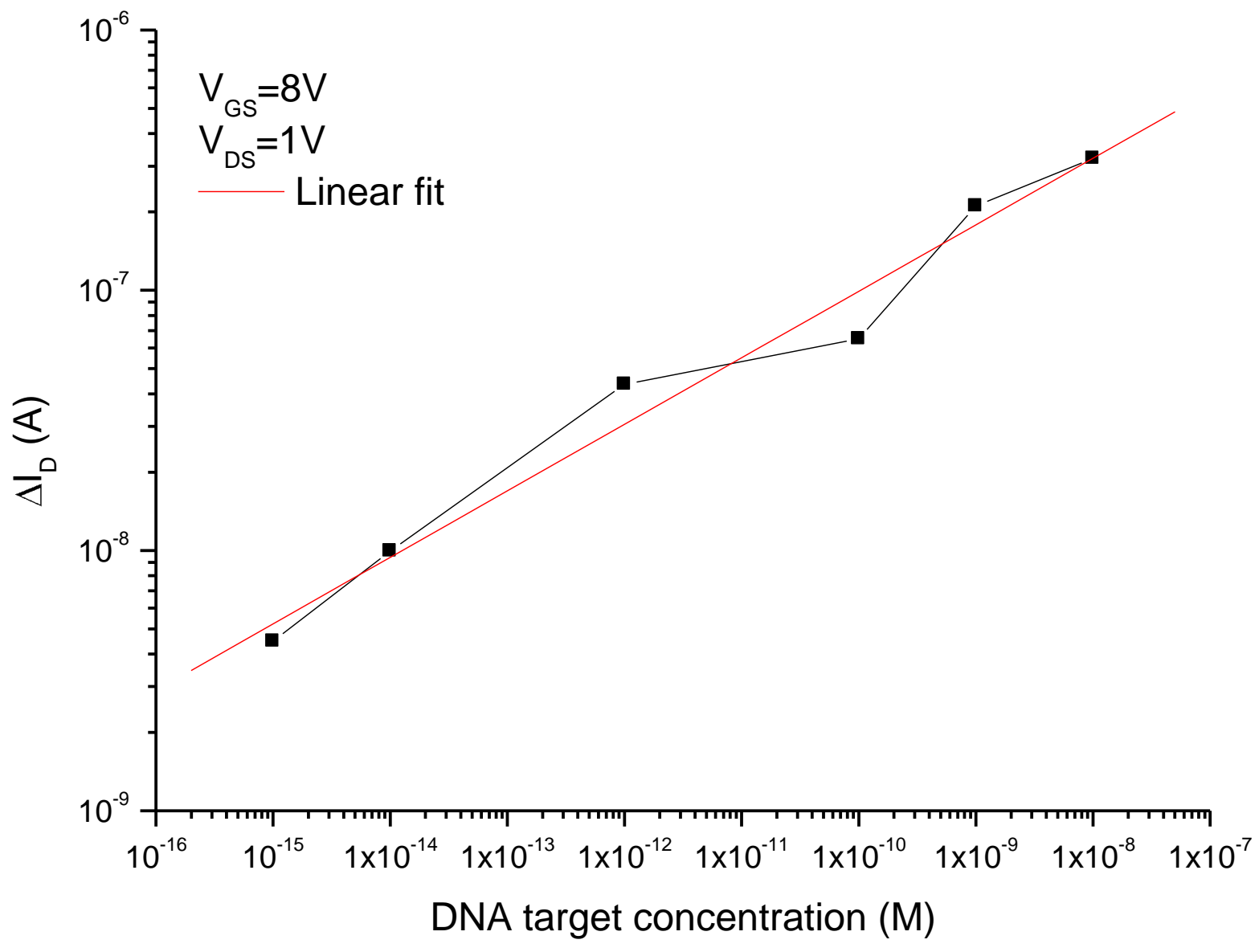

Fig. 8 